\title{
Krill oil, vitamin D and Lactobacillus reuteri cooperate to reduce gut inflammation
}

\author{
M. Costanzo ${ }^{1}$, V. Cesi $^{2}$, F. Palone ${ }^{1}$, M. Pierdomenico ${ }^{1}$, E. Colantoni ${ }^{1}$, B. Leter ${ }^{1}$, R. Vitali ${ }^{2}$, A. Negroni ${ }^{2}$, S. Cucchiara ${ }^{1}$ \\ and L. Stronati ${ }^{*}$ \\ ${ }^{1}$ Department of Pediatrics and Infantile Neuropsychiatry, Pediatric Gastroenterology and Liver Unit, Sapienza University of \\ Rome, Viale Regina Elena 324, 00161 Rome, Italy; ${ }^{2}$ Division of Health Protection Technologies, Territorial and Production \\ Systems Sustainability Department, ENEA, Via Angullarese 301, 00123 Rome, Italy; ${ }^{3}$ Department of Cellular Biotechnology \\ and Hematology, Sapienza University of Rome, Viale Regina Elena 324, 00161 Rome, Italy; laura.stronati@uniroma1.it
}

Received: 5 June 2017 / Accepted: 15 November 2017

(c) 2018 Wageningen Academic Publishers

RESEARCH ARTICLE

\begin{abstract}
Current research into original therapies to treat intestinal inflammation is focusing on no-drug therapies. KLD is a mixture of krill oil (KO), probiotic Lactobacillus reuteri (LR), and vitamin D (VitD3). The aim of this study was to assess in vitro and in vivo the potential cooperative effects of KLD in reducing gut inflammation. Colorectal adenocarcinoma cell lines, CACO2 and HT29, and C57BL/6 mice were used for in vitro and in vivo analyses, respectively. Cells were exposed to cytomix (interferon gamma + tumour necrosis factor alpha (TNF- $\alpha$ )) to induce inflammation or co-exposed to cytomix and KO, LR and VitD3 alone or to cytomix and KLD. Animals were treated for 7 days with dextran sodium sulphate (DSS) to induce colitis or with DSS and KLD. In vitro assays: F-actin expression was analysed by immunofluorescence; scratch test and trans-epithelial electric resistance test were performed to measure wound healing; adhesion/invasion assays of adhesive and invasive Escherichia coli (AIEC) bacteria were made; mRNA expression of TNF- $\alpha$, interleukin (IL)- 8 and vitamin D receptor (VDR) was detected by quantitative PCR. In vivo assays: body weight, clinical score, histological score and large intestine weight and length were estimated; mRNA expression of TNF- $\alpha$, IL-1 $\beta$, IL-6, IL-10 by quantitative PCR; VDR expression was detected by quantitative PCR and immunohistochemistry. In vitro: KLD restores epithelial cell-cell adhesion and mucosal healing during inflammation, while decreases the adhesiveness and invasiveness of AIEC bacteria and TNF- $\alpha$ and IL-8 mRNA expression and increases VDR expression. In vivo: KLD significantly improves body weight, clinical score, histological score and large intestine length of mice with DSS-induced colitis and reduces TNF- $\alpha$, IL- $1 \beta$ and IL-6 mRNA levels, while increases IL-10 mRNA and VDR levels. KLD has significant effects on the intestinal mucosa, strongly decreasing inflammation, increasing epithelial restitution and reducing pathogenicity of harmful commensal bacteria.
\end{abstract}

Keywords: intestinal inflammation, omega-3, probiotics, vitamin D, AIEC, DSS-mice

\section{Introduction}

Several observations from human patients as well as inflammatory rodent models have proven that microbiome is a central driver of chronic gut inflammation (Hirata et al., 2016; Wehkamp and Frick, 2016). Perturbations of the commensal microbiota, termed dysbiosis, are commonly observed in gut diseases, where promote mucosal inflammation through an imbalance between the protective bacterial species and the most dangerous ones (Von Martels et al., 2017). This explains the increasing use of live bacteria (probiotics) as a useful strategy to manipulate the microbiome improving the dysbiosis in patients with intestinal inflammation (Grimm and Riedel, 2016; Sánchez et al., 2016). Interestingly, probiotics have been recently used to reduce features associated with adherent invasive Escherichia coli (AIEC) bacteria that have been found greatly increased in patients with inflammatory bowel diseases (IBD) (Huebner et al., 2011; Rolhion and Darfeuille-Michaud, 2007). 
The composition of gut microbiota is deeply influenced by diet, one of the most influential lifestyle factors contributing to the rise of autoimmunity as well as inflammatory diseases, in both developed and developing countries (Albenberg and Wu, 2014; Dolan and Chang, 2016). In particular, the spread of the 'Western' diet, high in fat and protein but low in fruits and vegetables, may be associated with the increase of many diseases, such as obesity, type 2 diabetes, IBD, and food allergies (Hou et al., 2011; Kumari and Kozyrskyj, 2017; Uranga et al., 2016). The typical Western diet has a much greater ratio of $n-6$ polyunsaturated fatty acids (PUFAs), which display proinflammatory and immunoactive functions, compared with n-3 PUFAs, showing anti-inflammatory properties (Simopoulos, 2016; Wall et al., 2010). Recent evidence suggest that increasing the dietary intake of n-3 PUFAs (e.g. eicosapentaenoic acid (EPA) and docosahexaenoic acid (DHA)), through consumption of fatty fish or fish-oil supplements, may reduce the incidence of many chronic diseases that involve inflammatory processes (Jiang et al., 2016).

A number of studies have also consistently revealed an association between vitamin D deficiency and the occurrence of intestinal malabsorption syndromes, including cystic fibrosis, celiac disease, short bowel syndrome and IBD while vitamin D supplementation has been associated with decreased colitis disease activity and/ or alleviated symptoms in patients (Margulies et al., 2015; White, 2016; Zhang et al., 2013).

Previously, we showed that the use of krill oil (KO), a marine derived oil rich in phospholipids, the antioxidant astaxanthin and omega-3 fatty acids, is able to decrease intestinal inflammation (Costanzo et al., 2016). Afterwards, we showed that the use of DHA plus vitamin D treatment improves features of non-alcoholic fatty liver disease in children with serum vitamin D deficiency (Della Corte et al., 2016).

In the present paper, we aim to assess in vitro and in vivo the potential cooperative effects of a mixture (KLD) of KO, the probiotic Lactobacillus reuteri (LR), and vitamin D in down-regulating gut inflammation by improving mucosal healing and epithelial barrier permeability as well as limiting pathogenicity of harmful intestinal bacteria.

\section{Material and methods}

\section{KLD components}

KO (Giellepi SPA, Milan, Italy), whose specific composition is phospholipids $(51 \%, \mathrm{w} / \mathrm{w})$, DHA $(7 \%, \mathrm{w} / \mathrm{w})$, EPA $(12 \%$, w/w), omega-3 (-3, or PUFA-3) $(21 \%, w / w)$, astaxanthin $(0.04 \%, w / w)$. The manufacturer supplied a certificate of analysis for the use in nutritional supplements and functional food, which includes total of active ingredients, physical and chemical tests and microbiological tests.

VitD3 (Giellepi SPA), whose specific name is 25-diidrossivitamin $\mathrm{D} 3\left(1,25(\mathrm{OH})_{2} \mathrm{D} 3\right)$. The manufacturer supplied a certificate of analysis for the use in nutritional supplements and functional food, which includes total of active ingredients, physical and purity tests.

LR (BioGaia, Stockholm, Sweden) was cultured in De Man, Rogosa and Sharpe (MRS; Sigma-Aldrich, Buchs, Switzerland) agar plates for $24 \mathrm{~h}$ at $37{ }^{\circ} \mathrm{C}$ and then subcultured in MRS Broth with overnight incubation at $150 \mathrm{rpm}, 37^{\circ} \mathrm{C}$. The manufacturer supplied a certificate of analysis for the use in nutritional supplements and functional food, which includes quality control tests and microbiological specifications.

KLD mixture used for in vitro experiments consists of: $\mathrm{KO}[0.25 \mathrm{mg} / \mathrm{ml}]+\mathrm{VitD} 3[100 \mathrm{nM}]+\mathrm{LR}\left[1 \times 10^{9} \mathrm{cfu} / \mathrm{ml}\right]$ in eukaryotic cell growth media (DMEM or McCoy's $5 \mathrm{~A}$ ); for in vivo experiments concentrations were: $\mathrm{KO}[0.8 \mathrm{mg} /$ $\mathrm{gr}]+$ VitD3 $\left[2.5 \times 10^{-5} \mathrm{mg} / \mathrm{gr}\right]+\mathrm{LR}\left[5 \times 10^{7} \mathrm{cfu} / \mathrm{gr}\right]$ pro die in phosphate buffered saline (PBS); $0.2 \mathrm{ml}$ of KLD was administered by gavage twice daily.

This specific ratio and combination were chosen as follows: $\mathrm{KO}$ : according to the allometric scaling laws (West and Brown, 2005) and since DHA+EPA are 20\% in KO, by administering $16 \mathrm{mg} / \mathrm{mouse}(0.8 \mathrm{mg} / \mathrm{gr})$ pro die, the protocol is in compliance with the EFSA note released in July 2012, which fixes the safe daily intake of DHA+EPA in 2 to 4 gr per day (3.2 $\mathrm{mg}$ of omega-3 in mouse are equivalent to $2 \mathrm{gr}$ in humans); VitD3: $2.5 \times 10^{-5} \mathrm{mg} / \mathrm{gr}$ pro die is equivalent to $0.5 \mu \mathrm{g}$ per mouse per day which is the higher dose described for in vivo studies (Meeker et al., 2016); LR: $5 \times 10^{7} \mathrm{cfu} / \mathrm{gr}$ of LR is equivalent to $1 \times 10^{9} \mathrm{cfu}$ pro die that is the dose used for in vivo studies (Zheng et al., 2016).

\section{Adherent invasive Escherichia coli strain LF82}

The AIEC strain LF82 (ileal Crohn's strain, kindly provided by Prof. Arlette Darfeuille-Michaud, Clermont Université, Université d'Auvergne, Clermont-Ferrand, France) was cultured in MacConkey agar plates for $24 \mathrm{~h}$ at $37^{\circ} \mathrm{C}$ and then sub-cultured in Luria Bertani Broth (LB; Oxoid, Basingstoke, UK) with overnight incubation at $150 \mathrm{rpm}$, $37^{\circ} \mathrm{C}$.

\section{Cell lines}

Human colorectal adenocarcinoma cell lines, CACO2 and HT29, were obtained from the American Type Culture Collection (ATCC, Rockville, MA, USA). Cells were grown at confluence at $37^{\circ} \mathrm{C}$ in Dulbecco's minimum essential 
medium (DMEM; Gibco, Life Technologies, Carlsbad, CA, USA) and McCoy's 5A medium (Gibco), respectively, supplemented with $10 \%$ inactivated foetal calf serum (FCS; Euroclone, Milan, Italy) and $2 \mathrm{mM} \mathrm{L}$-glutamine, $100 \mathrm{U} / \mathrm{ml}$ penicillin and $100 \mathrm{~g} / \mathrm{ml}$ streptomycin (Biochrom, Berlin, Germany).

Confluent CACO2 or HT29 cells were grown in absence or presence of cytomix (tumour necrosis factor alpha (TNF- $\alpha$ ) + interferon gamma: $10 \mathrm{ng} / \mathrm{ml}+250 \mathrm{ng} / \mathrm{ml}$; Sigma) to induce inflammation or co-exposed to cytomix and KO, LR and VitD3 singularly or to cytomix and KLD for 24-48 h or in presence of the AIEC reference strain, LF82 (see Bacterial Adhesion and Invasion Assay) or LF82 and KLD, for $3 \mathrm{~h}$.

\section{Scratch test}

Wound healing was assessed by the scratch test as previously described (Costanzo et al., 2016). Briefly, confluent HT29 cells were seeded on to a 2 well cell-culture-slide at a density of $2 \times 10^{5}$ cells $/ \mathrm{ml}$. The linear scratch was made with a 10 microliter sterile pipette tip and the gap widths $(1 \mathrm{~mm}$ at day 0 ) were measured after 24 and $48 \mathrm{~h}$. Cells were exposed to the cytomix to induce inflammation or co-exposed to cytomix and KLD for 24 and $48 \mathrm{~h}$. Scratch wound healing was treated with haematoxylin and eosin staining protocol (H\&E) and then observed under a Nikon Eclipse 80i digital microscope (Nikon, Tokyo, Japan) at 10× magnification.

\section{Immunofluorescence}

Cells exposed to the cytomix or co-exposed to cytomix and KLD or to cytomix and single components (KO, VitD3, LR) were grown at confluence on a microscope glass slide for $24 \mathrm{~h}$. Then, cells were fixed for $10 \mathrm{~min}$ in PBS with 4\% paraformaldehyde. Cells were washed in PBS, permeable by incubation for $10 \mathrm{~min}$ in PBS-0.1\% Triton X-100 (Sigma), and then blocked for $30 \mathrm{~min}$ in PBS-1\% BSA. For F-actin staining, samples were incubated with Alexa Fluor 488-conjugated Phalloidin (1:50 dilution; Invitrogen, Carlsbad, CA, USA) according to manufacturer's instructions. 4',6-diamidino-2-phenylindole was added for nuclei counterstaining.

\section{Bacterial adhesion and invasion assay}

\section{Adhesion assay}

CACO2 and HT29 cells were grown on 24-well plates at confluence and infected with LF82 strains at a multiplicity of infection (MOI) of 10:1 for CACO2 and 100:1 for HT29, at $37^{\circ} \mathrm{C}$ for $3 \mathrm{hrs}$. To quantify the adherence of LF82, infected cells were washed twice in PBS and lysed for $10 \mathrm{~min}$ with 0.5 $\mathrm{ml}$ of $0.1 \%$ Triton X-100 in PBS buffer. Adherent bacteria were recovered and plated on LB agar plates. The latter were incubated at $37^{\circ} \mathrm{C}$ overnight and then the colonies were counted for statistical analysis.

\section{Invasion assay}

CACO2 and HT29 were infected and incubated as above. After incubation, cells were washed twice in sterile PBS and then incubated in DMEM and McCoy's medium, respectively with $0.1 \mathrm{mg} / \mathrm{ml}$ gentamicin for $1 \mathrm{~h}$ to kill extracellular bacteria. Cells were washed twice in sterile PBS. Lysis, incubation and counts were performed as in the adhesion assay. To ensure maximum reproducibility, accuracy and statistical significance, adhesion and invasion assays were carried out simultaneously in triplicates.

\section{Quantitative PCR analysis}

Cytokine expression was detected by quantitative PCR. Primers were designed to non-redundant sequences using Primer Express v. 3.0 (Applied Biosystems, Foster City, CA, USA). RNA purity was assessed by $260 / 280$ and $260 / 230 \mathrm{~nm}$ absorbance ratio (2.0 or above). RNA integrity was checked by agarose-formaldehyde analysis. Total RNA $(1 \mu \mathrm{g})$ was reverse- transcribed to cDNA by a High Capacity cDNA Reverse Transcription Kit (Applied Biosystems). Quantitative PCR amplification was done with an ABI PRISM 7300 Sequence Detection System using the SYBR Green kit (Applied Biosystems), according to the manufacturer's instructions.

The following primers were used (Table 1). Fold of induction is the ratio between observed genes and endogenous control expression levels made in triplicate.

\section{Trans-epithelial Electric Resistance (TEER) assay}

CACO2 cells were grown at $37^{\circ} \mathrm{C}$ in $5 \% \mathrm{CO}_{2}$ on polyethylene terephthalate membrane inserts, pore size $0.4 \mathrm{~mm}$ (Falcon; Becton Dickinson, Franklin Lakes, NJ, USA) in DMEM supplemented with 10\% FCS. TEER values were measured using a Millicell-ERS voltohmmeter (Millipore, Billerica, MA, USA) according to the manufacturer's instructions. Electrodes were sterilised by immersion in $70 \%$ ethanol, then they were washed in sterile PBS prior to use. Calculations for $\mathrm{Xcm}^{2}$ were made by subtracting values of blank inserts from all samples and multiplying by the area seeded with cells. Subtracting filter resistance, epithelial mono-layers were grown to an average TEER of about $700 \mathrm{Xcm}^{2}$ tissue monolayer. Cells were infected with the reference AIEC strain LF82 at MOI 10:1 and KLD (at established concentration), applied to the apical transwell compartment, at $37^{\circ} \mathrm{C}$ in $5 \% \mathrm{CO}_{2}$. Changes in TEER over time were determined every $2 \mathrm{~h}$ and triplicate TEER readings from three independent experiments were normalised, averaged, and expressed as percent change. 
Table 1. Primer sequences used for quantitative PCR.

\begin{tabular}{|c|c|c|}
\hline Gene $^{1}$ & $F / R^{2}$ & Primer \\
\hline \multirow[t]{2}{*}{ TNF-a } & $\mathrm{F}$ & 5'-TCTGGCCCAGGCAGTCAGATC-3' \\
\hline & $\mathrm{R}$ & 5'-CAGTGATGTTGGGGATAAAGAGC-3' \\
\hline \multirow[t]{2}{*}{ IL-8 } & $\mathrm{F}$ & 5'-ATGACTTCCAAGCTGGCCGTGGCT-3' \\
\hline & $\mathrm{R}$ & 5'-TCTCAGCССTСTTCAAAAАСTTCTC-3' \\
\hline \multirow[t]{2}{*}{ IL-1 $1 \beta$} & $\mathrm{F}$ & 5'-CGAGGCAGTATCACTCATTG-3' \\
\hline & $\mathrm{R}$ & 5-CGTTGCTTGGTTCTCCTTGT-3' \\
\hline \multirow[t]{2}{*}{ IL-6 } & $\mathrm{F}$ & 5'-CAAGTCGGAGGCTTAATTACACATG-3' \\
\hline & $\mathrm{R}$ & 5'AGAAAAGAGTTGTGCAATGGCA-3' \\
\hline \multirow[t]{2}{*}{ IL-10 } & $\mathrm{F}$ & 5'AACAAAGGACCAGCTGGACAAC-3' \\
\hline & $\mathrm{R}$ & 5'-GGCAACCCAAGTAACCCTTAAA-3' \\
\hline \multirow[t]{2}{*}{ VDR (cells) } & $\mathrm{F}$ & 5'-GGACTGCCGCATCACCAA-3' \\
\hline & $\mathrm{R}$ & 5'-ТСАТСТСССGСТTССТСТ-3' \\
\hline \multirow[t]{2}{*}{ VDR (mice) } & $\mathrm{F}$ & 5'-САССТGССССTTCAATGG-3' \\
\hline & $\mathrm{R}$ & 5'-GCAGTGTCGCCGGTTGTC-3' \\
\hline \multirow{2}{*}{$\begin{array}{l}\text { GAPDH (cells } \\
\text { and mice) }\end{array}$} & $\mathrm{F}$ & 5'-AACTTTGGCATTGTGGAAGG-3' \\
\hline & $\mathrm{R}$ & 5'-CACATTGGGGGTAGGAACAC-3' \\
\hline
\end{tabular}

${ }^{1}$ TNF- $\mathrm{a}=$ tumour necrosis factor alpha; IL = interleukin; VDR = vitamin $\mathrm{D}$ receptor; GAPDH = glyceraldehyde 3-phosphate dehydrogenase (household gene).

${ }^{2} \mathrm{~F}=$ forward; $\mathrm{R}=$ reverse.

\section{Animals}

C57BL/6 female mice ( 8 to 9 weeks of age) were purchased from the animal housing unit of ENVIGO, SRL. Animals were housed in collective cages at $22 \pm 1^{\circ} \mathrm{C}$ under a $12 \mathrm{~h}$ light/ dark cycle and with food and water provided ad libitum. Acute colitis was induced through administration of dextran sodium sulphate (DSS, molecular mass, 36,000-50,000 Da; MP Biomedicals, Santa Ana, CA, USA), dissolved in autoclaved drinking water, for 7 days. Mice, 5 for each group, were randomly divided into four experimental groups: the control group received regular drinking water (UN); KLD-treated group received two daily oral gavages of KLD (KLD); DSS-treated group was given a solution with $3 \%(\mathrm{w} / \mathrm{v})$ DSS (DSS); fourth group received a combined treatment with 3\% DSS and two daily oral gavages of KLD (DSS+KLD). Mice were daily checked for behaviour, body weight, stool blood and consistency.

The experimental procedures were previously approved by the Ministry of Health for the protection of animals used for experimental purposes, and the study was conducted in accordance with Italian regulations on animal welfare. The protocol was approved by the Committee on the Ethics of Animal Experiments of the Italian National Agency for New Technology, Energy and Sustainable Economic Development (ENEA) (Permit Number: 76/2017-PR).

\section{Assessment of DSS-induced colitis and histological score}

All animals were daily examined and the clinical score (CS) was assessed according to the criteria of Maxwell et al. (Maxwell et al., 2009) by assessing stool consistency (0 for normal stool, 1 for moist/sticky stool, 2 for soft stool, 3 for diarrhoea), presence of blood in stool ( 0 for no blood, 1 for evidence of blood in stool or around anus, and 2 for severe bleeding) and general appearance of the animal (0 was assigned if normal, 1 for ruffled fur or altered gait, 2 for lethargic or moribund). Mice were daily weighed, and the percentage of weight loss was calculated in relation to the starting weight using the formula: [(Weight on day X Initial weight) / Initial weight] $\times 100$ (Maxwell et al., 2009).

The $7^{\text {th }}$ day, animals were euthanised, colons removed and examined for weight, length (measured from the anus to the top of the caecum) and stool consistency. Distal colonic specimens were frozen in liquid nitrogen or fixed immediately in a $10 \%(\mathrm{w} / \mathrm{v})$ formalin solution for further analyses.

For histological analysis, fixed colonic tissues were embedded in paraffin, sectioned ( $4 \mu \mathrm{m}$ thickness), mounted on glass slides, and deparaffinised. Slices were stained using standard haematoxylin and eosin (H\&E) techniques. Samples were analysed by light microscopy and were scored according to previous procedure. (Maxwell et al., 2009) (0 for normal; 1 for slight increase in cellularity (primarily lymphocytes in the lamina propria); 2 for increase in cellularity, neutrophils present, mild oedema; 3 for diffuse increase in cellularity, focal erosions or ulcerations of mucosa; 4 increased cellularity, large and or multifocal mucosal ulcerations; 5 for diffuse ulceration, loss of mucosal architecture). Experiments were double-blind.

\section{Immunohistochemistry}

Sections $(4 \mathrm{~mm})$ of paraffin-embedded intestinal colonic samples from mice were prepared following the standard protocol. Briefly, sections were dewaxed for $20 \mathrm{~min}$ at $56{ }^{\circ} \mathrm{C}$ and incubated in ab973 buffer $\mathrm{pH} 6.0$ for 20 min at $95^{\circ} \mathrm{C}$. Afterward, sections were washed in water for $30 \mathrm{~min}$ and peroxidases inhibited by incubation in $3 \% \mathrm{H}_{2} \mathrm{O}_{2}$ for $15 \mathrm{~min}$. Sections were treated with Rodent Block for $50 \mathrm{~min}$ and incubated with primary anti-VDR (D-6) antibody (SantaCruz Biotechnologies, Dallas, TX, USA), diluted 1:50 in phosphate-buffered saline over night at $4{ }^{\circ} \mathrm{C}$ in a moist chamber. Then, they were washed in TBS-T $0.1 \%$, incubated for $15 \mathrm{~min}$ with Mouse on Mouse HRP Polymer (ab127055 - Mouse on Mouse Polymer IHC Kit; Abcam, Cambridge, UK), and washed again TBS-T 0.1\%. The DAB detection kit (DAKO; Abcam) was used, as suggested by the providers, to visualise the antigen. Finally, samples were stained with $H \& E$. 


\section{Statistics}

Data are given as mean \pm standard deviation. All experiments in vitro were repeated three times. Comparison between groups was performed by a two-tailed Student t-test (significance taken as $P<0.05$ ). In vivo experiments are the mean of five observations per group. Comparison between groups was performed by non-parametric test (Mann-Whitney) (values of $P<0.05$ were considered significant).

\section{Results}

\section{KLD restores epithelial cell-cell adhesion and tissue architecture altered by cytomix-induced inflammation}

F-actin localisation and expression were determined by immunofluorescence in $\mathrm{CACO} 2$ cells. F-actin filaments, components of the actin cytoskeleton that in eukaryotes are essential to junctional assembly, are organised into stress fibres (SFs) that are known to increase in number and thickness during inflammation. Results showed that exposure to cytomix caused a sharp increase in SFs numbers with a disorganisation in junctional assembly in all samples compared to untreated cells. However, KLD promoted a proper tissue re-organisation strongly reducing SFs, better than single components (Figure 1).

It is worth noting that LR, despite having a well-known role in preventing and/or treating gastrointestinal diseases (Cruchet et al., 2015), minimally alters the morphology of intestinal barrier (Figure 1), albeit much less than other commensal species, like the AIEC reference strain LF82 (Supplementary Figure S1A). Nevertheless, the combination of LR with VitD3 and KO (KLD) completely abolished this effect. Interestingly, KLD did not change the vitality of LR in both cell lines (CACO2, HT29) after 24 h (Supplementary Figure S1B).

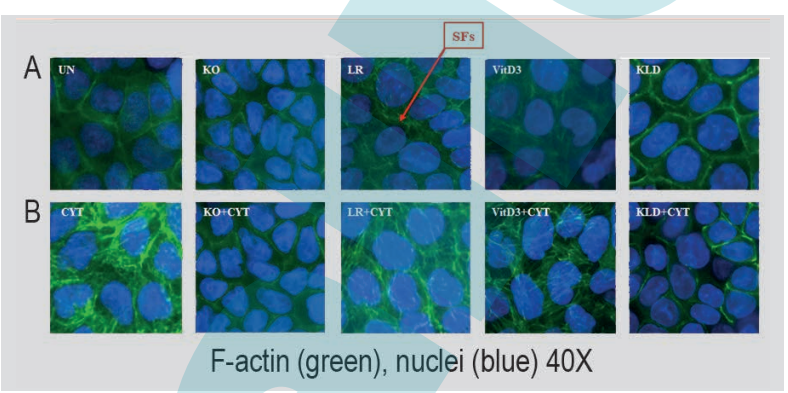

Figure 1. Cytomix induces stress fibres (SFs), but KLD reduces SFs formation better than single components. UN = untreated; $\mathrm{KO}=$ krill oil; $\mathrm{LR}=$ Lactobacillus reuteri; VitD3 = vitamin D3; $K L D=K O+L R+V i t D 3 ; C Y T=$ cytomix $(T N F-\alpha+I F N-\gamma)$.

\section{KLD strongly inhibits adhesiveness and invasiveness of AIEC bacteria}

KLD significantly decreased adhesion as well as invasion of LF82 in CACO2 and HT29 cells $(P<0.01$ or $P<0.05$, respectively) (Figure 2A). Accordingly, KLD significantly improved cell morphology of the epithelial barrier by decreasing SFs (Figure 2B) and increasing the TEER (Figure $2 C)$, both deeply altered by LF82 exposure. LR was found to be more effective in reducing adhesiveness as well as invasiveness of AIEC bacteria when administered in KLD formulation (reduction of around 75 and 64\%, respectively) (Figure 2A) than alone (reduction of around $50 \%$ and $40 \%$, respectively) (Supplementary Figure S2).

\section{KLD decreases mRNA levels of IL-8 and TNF- $\alpha$ and increases mRNA expression of VDR during inflammation}

KLD significantly reduced IL- 8 and TNF- $\alpha$ mRNA levels in CACO2 and HT29 cells $(P<0.01)$ as much as $\mathrm{KO}$ and VitD3 alone, however, it was able to strengthen the anti-inflammatory potential of LR as compared to LR administered alone (Figure 3). Additionally, KLD significantly increased the expression level of vitamin D receptor $(\mathrm{VDR})(P<0.01)$ (Figure 3$)$.

\section{KLD significantly improves mucosal healing during cytomix-induced inflammation}

Scratched cells exposed to cytomix showed a delay of $\sim 9 \%$ and $\sim 26 \%$ of wound healing (WH) as compared to unexposed cells ( 24 h: $59.39 \pm 11.82$ vs $68.32 \pm 9.32 \%, P<0.01$; 48 h: $35.41 \pm 8.72$ vs $61.49 \pm 7.45 \%, P<0.001)$. However, cotreatment with KLD significantly improved the rate of $\mathrm{WH}$ when compared to cytomix at $24(+15 \%)$ and $48 \mathrm{~h}(+33 \%)$ $(68.32 \pm 9.32$ vs $52.81 \pm 8.23 \%, P<0.01$ and $61.49 \pm 7.45$ vs $27.95 \pm 7.81 \%, P<0.01$, respectively) (Figure 4, Supplementary Figure S3).

\section{KLD significantly reduces the severity of DSS-induced colitis in mice}

After DSS treatment of mice and induction of severe colitis, results showed that animals treated with DSS + KLD had a statistically significant recovery of body weight $(P<0.01)$, CS $(P<0.01)$, and colon length $(P<0.01)$, as compared to DSStreated animals (Figure 5A, B and C). Anti-inflammatory effects of KLD were further confirmed by the histological analysis which showed an important recovery of the morphological features of the intestinal mucosa and a significant reduction of the histological score $(P<0.05)$ (Figure 5D and E). 


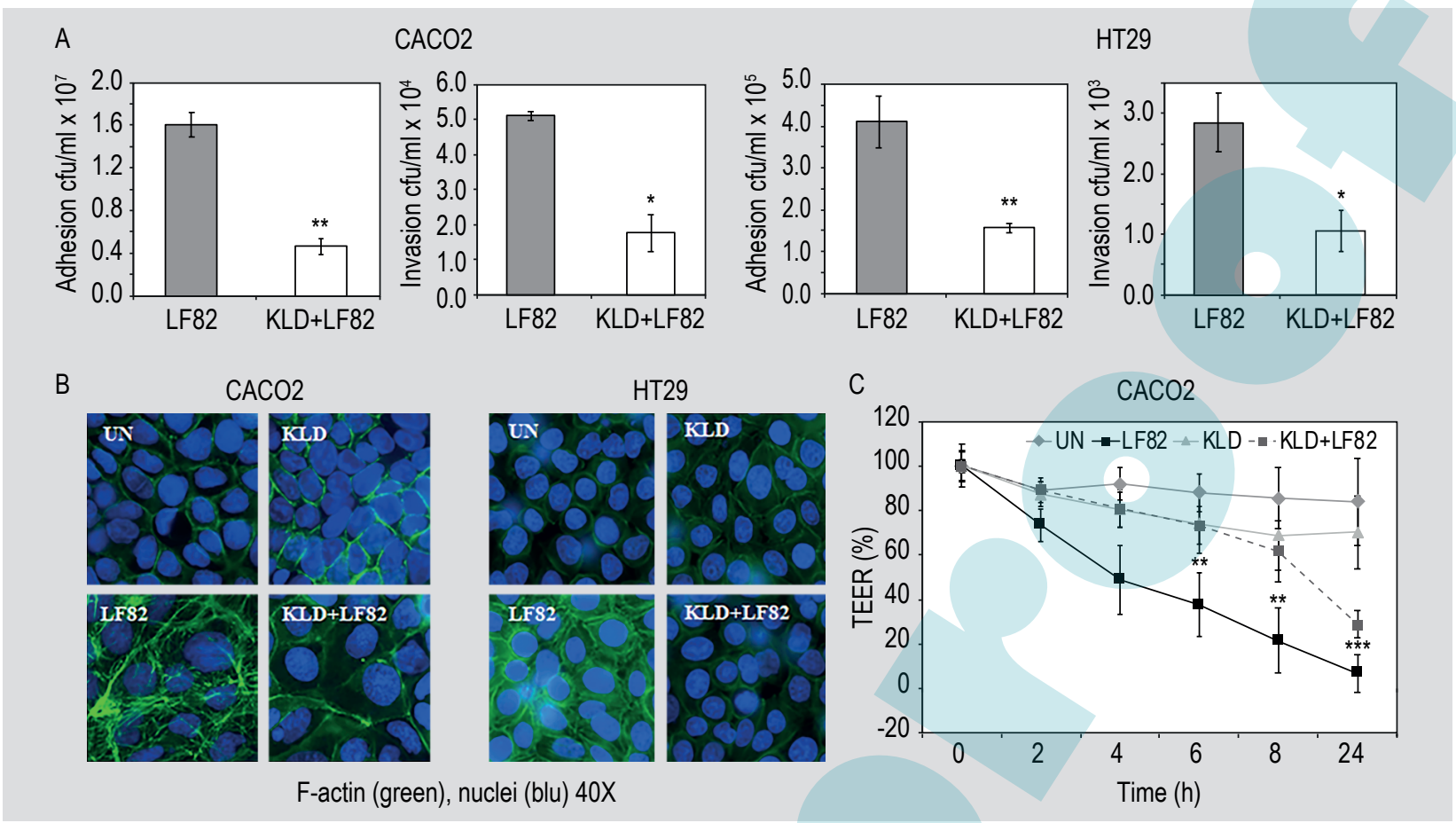

Figure 2. (A) Effects of KLD on adhesion and invasion of adherent invasive Escherichia coli strain LF82. (B) Effect of KLD on epithelial architecture: SFs are induced by LF82 but reduced by KLD in two different cell lines (CACO2 and HT29); (C) Trans Epithelial Electric Resistance (TEER) is reduced after infection with LF82, but recovered by KLD (black vs red lines). * $P<0.05$; ** $P<0.01 ;{ }^{* * *} P<0.001$. UN = untreated; KLD = krill oil + Lactobacillus reuteri + vitamin D3.

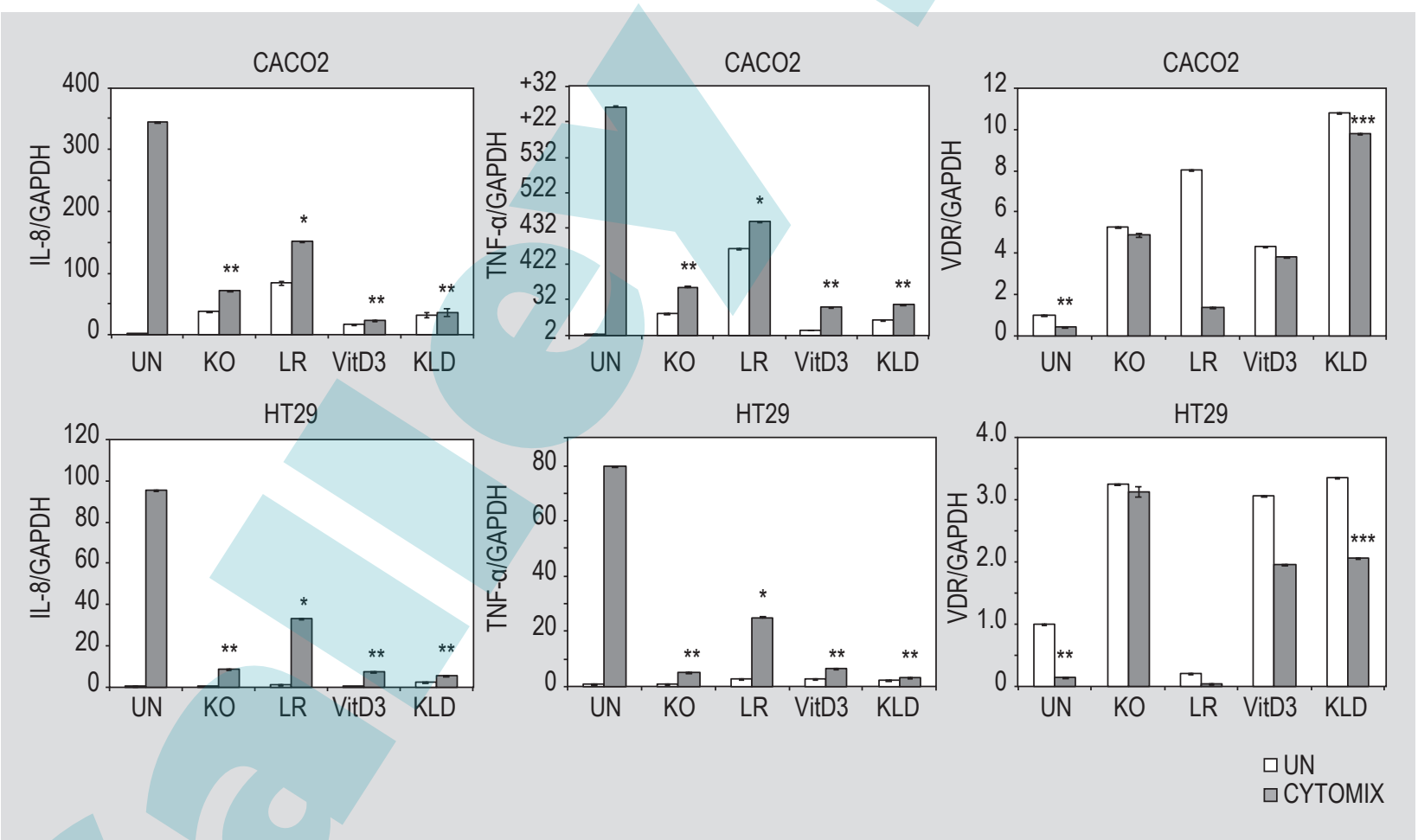

Figure 3. Effects of KLD on mRNA expression of pro-inflammatory cytokines interleukin (IL)-8 and tumour necrosis factor alpha (TNF-a), and vitamin D receptor (VDR) in CACO2 and HT29. UN = untreated; $K O=$ krill oil; LR = Lactobacillus reuteri; VitD3 = vitamin $D 3 ; K L D=K O+L R+$ VitD3; cytomix $=$ TNF- $\alpha+$ interferon gamma. ${ }^{*} P<0.05 ;{ }^{* *} P<0.01 ;{ }^{* * *} P<0.001$. 


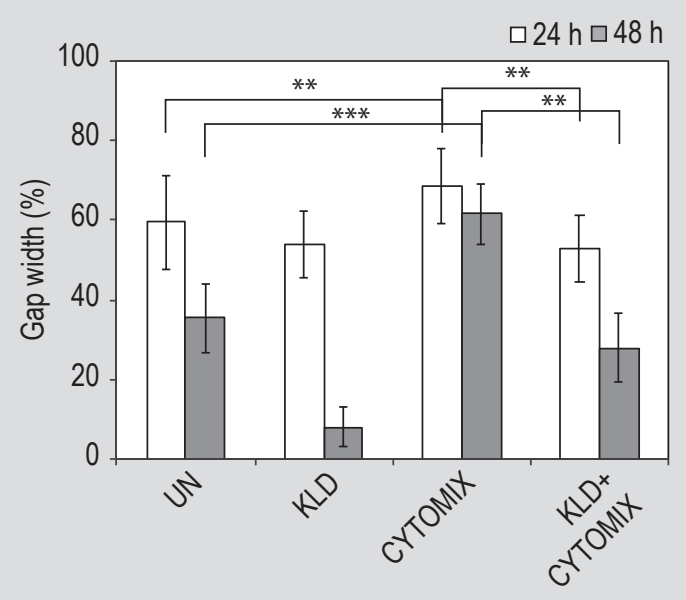

Figure 4. The effect of KLD on mucosal healing evaluated by scratch test. The width at time $0 \mathrm{~h}$ was taken as $100 \%$. UN = untreated; KLD = krill oil + Lactobacillus reuteri + vitamin D3; cytomix: tumour necrosis factor alpha + inferno gamma. ** $P<0.01$; *** $P<0.001$.

Moreover, tissue samples taken from the large colon of mice were analysed to assess the mRNA expression of the pro-inflammatory cytokines, TNF $\alpha$, IL-1 $\beta$ and IL-
6, the anti-inflammatory cytokine IL-10 and the VDR. Results showed a dramatic decrease of TNF $\alpha$, IL-1 $\beta$, IL-6 $(P<0.001)$ as well as a strong increase of IL-10 and VDR ( $P<0.001$ and $P<0.01$, respectively) in mice treated with DSS+KLD as compared to mice treated with DSS (Figure 6). To confirm the triggering of vitamin D signalling, the VDR expression was also confirmed by immunohistochemistry. Results showed that VDR levels were severely reduced after DSS treatment, while exposure to KLD restored a proper condition (Figure 7).

\section{Discussion}

Much evidence supports the view that the lack of gut barrier integrity is strongly associated to gastrointestinal as well as extra-intestinal diseases (König et al., 2016; Mittal and Coopersmith, 2014; Odenwald and Turner, 2017). Thus, targeting and restoring the epithelial barrier is a tempting therapeutic goal and agents usable in this sense are presently highly desirable. This is especially true for intestinal chronic inflammatory entities, like IBD, that are becoming an emergency in developed and developing countries (Ye et al., 2015).

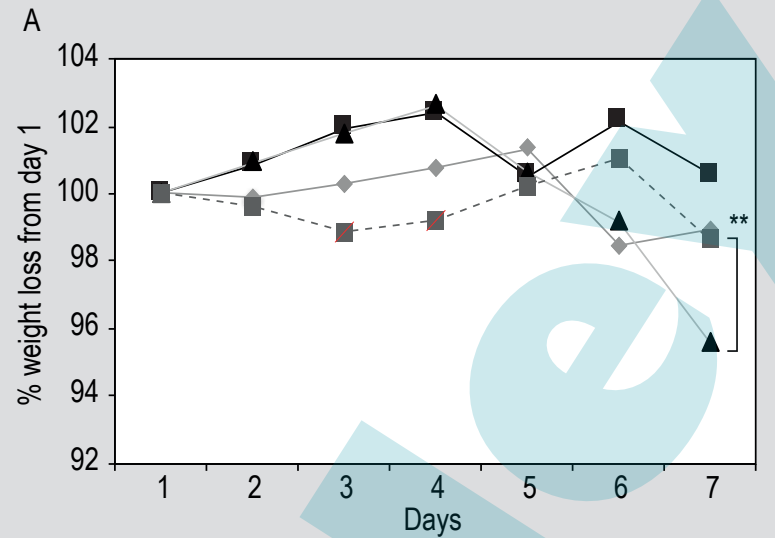

C

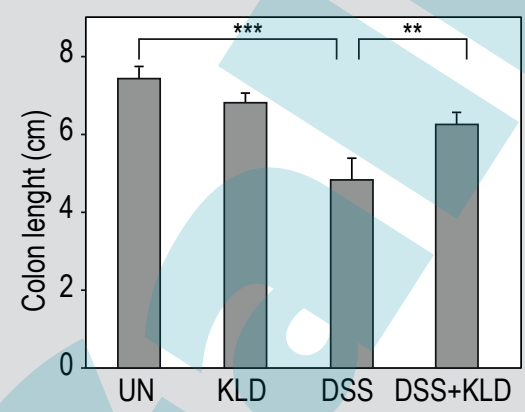

D UN

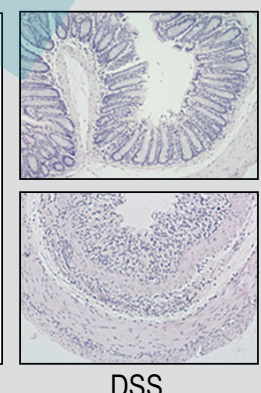

B

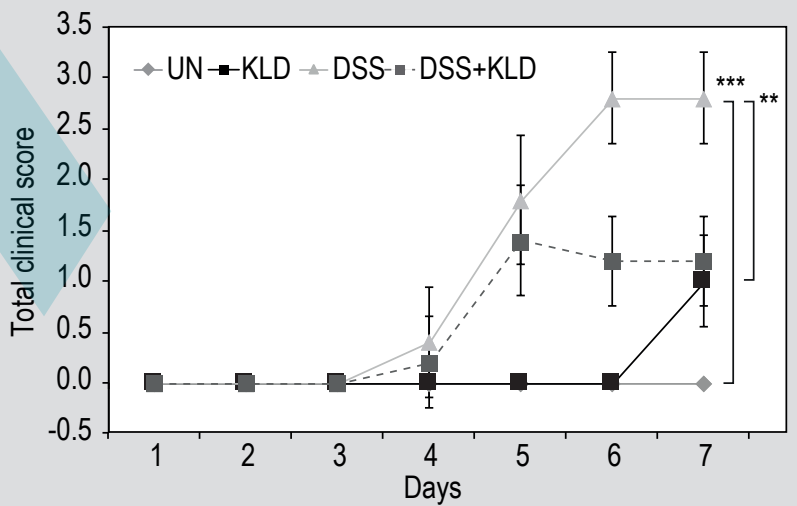

KLD

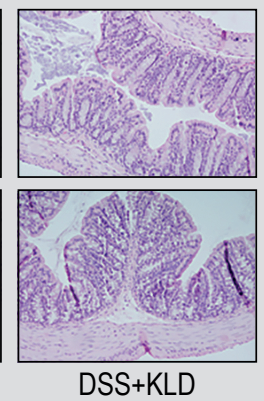

$\mathrm{E}$

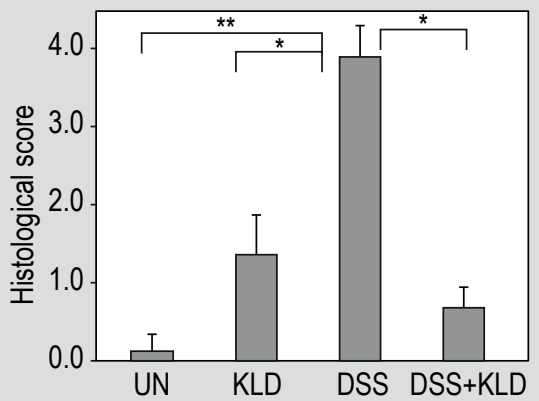

Figure 5. Effects of KLD on DSS-induced colitis in mice: (A) weight loss; (B) clinical score; (C) colon length; (D) histology; (E) histological score. UN = untreated; KLD = krill oil + Lactobacillus reuteri + vitamin D3. * $P<0.05 ;{ }^{* *} P<0.01$; ${ }^{* *} P<0.001$. 

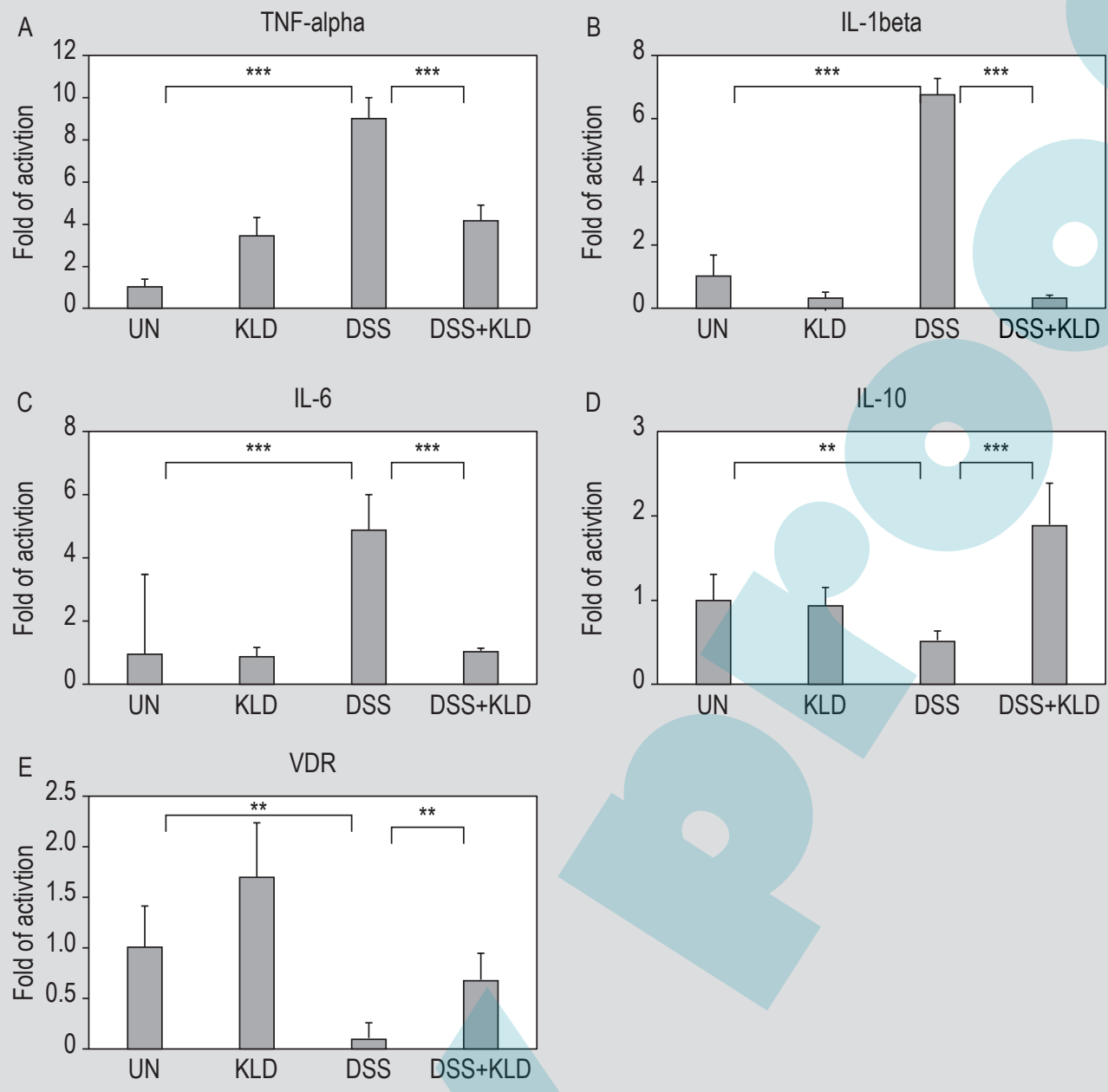

Figure 6. mRNA expression of (A) tumour necrosis factor alpha (TNF- $\alpha$ ), (B) interleukin (IL)-1 $\beta$, (C) IL-6, (D) IL-10 and (E) vitamin $D$ receptor (VDR) in DSS-induced colitis mice. UN = untreated; KLD = krill oil + Lactobacillus reuteri + vitamin D3. * $P<0.05$; * $P<0.01 ;{ }^{* * *} P<0.001$.

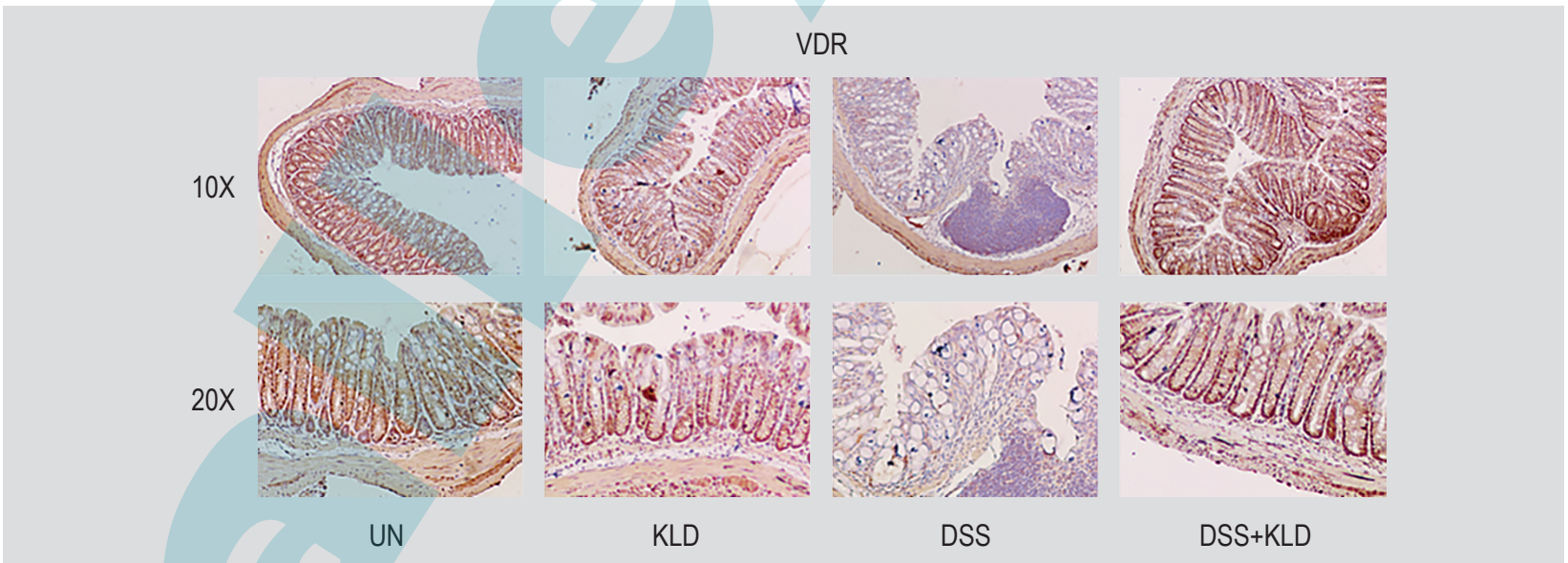

Figure 7. Histochemical staining of vitamin $D$ receptor in colon tissue of DSS-induced colitis mice. UN = untreated; KLD = krill oil + Lactobacillus reuteri + vitamin D3. 
IBD is characterised by defects in the intestinal epithelial barrier as well as imbalance of the intestinal microbiota, resulting in active inflammation. These diseases are usually managed with immunomodulators, corticosteroids or, more recently, biological agents that, next to their therapeutic action, have important side effects of toxic nature, often harmful to the health of patients. Hence, current research into original therapies to treat intestinal inflammation is focusing on no-drug therapies.

Studies to date reveal that vitamin D signalling has widespread effects on gut homeostasis, (Dimitrov and White, 2017). Vitamin D elicits its effects through binding to the VDR, a transcription factor for genes with promoters containing vitamin D response elements (VDREs). Strategies to restore VDR expression in inflamed mucosa may be important for preventing and treating IBD and other illness (Shang and Sun, 2017; Wu et al., 2010, 2015). As well, relevant data are deriving from studies on probiotics as selective modulators of intestinal microbiota. In particular, remodelling microbiota with LR reduced inflammation in animal models and humans (He et al., 2017; Oliva et al., 2012; Thomas et al., 2016). In our study, we observed that LR itself may produce a mild increase in mucosal inflammation, as shown by the increase in SFs number and pro-inflammatory cytokine expression. This event, also reported by other authors (Liu et al., 2010; Mangalat et al., 2012), is attributable to the fact that $L R$ is still a bacterium and, as such, somehow stimulates the immune system, although much less than other more harmful commensal species, like the AIEC bacteria (as also shown by our data). The combination with KO and VitD3 abrogates this effect, while maintaining unaltered the properties of the probiotic. Indeed, our results show that, within KLD, LR shows the same vitality as when it is grown in standard medium and, more interestingly, it is even more competitive with AIEC bacteria. This is extremely relevant since in different chronic inflammatory conditions, including IBD, AIEC strains are significantly increased and the use of probiotics may represent in the future a successful strategy to reduce their number and pathogenicity, as already suggested (Huebner et al., 2011).

Recent studies show the positive effects of omega-3 PUFA for the treatment of intestinal inflammation as they alleviate the symptoms and promote better mucosal integrity. Furthermore, some evidence show that omega- 3 fatty acids can reduce body weight gain and chronic inflammation through modulation of the gut microbiota (Kaliannan et al., 2016; Tabbaa et al., 2013).

Hence, it was born the idea to combine the $\mathrm{KO}$, that is rich in n-3 PUFAs, with VitD3, both deficient in patients with chronic intestinal inflammation, and LR, studying its potential to reduce intestinal inflammation by using a wide range of investigations. Indeed, we explored cell-cell adhesion and morphology of epithelial cells, both related to epithelial permeability and analysed the expression levels of pro-inflammatory cytokines, whose increase is strictly linked to inflammation. Moreover, we observed the resistance of the epithelial layer to the infectiveness of detrimental bacteria, typically increased during inflammation. Interestingly, our results show that KLD has significant effects on the intestinal mucosa, strongly decreasing inflammation, improving epithelial restitution and permeability, also strengthening vitamin $\mathrm{D}$ activity by enhancing VDR expression, as well as reducing harmfulness of some types of commensal bacteria.

We believe that this outcome is due to the fact that KLD is active on two fronts: on the one hand, it reduces the epithelial damage by improving barrier integrity and mucosal healing; on the other hand, it contributes to restore a condition of intestinal eubiosis by limiting the effectiveness of groups of pathobionts that increase during inflammation. Concurrently, KLD clearly lessens the aforementioned immunomodulatory effect caused by LR without modifying its beneficial properties. Interestingly, LR contrasts more effectively AIEC harmfulness when administered in KLD formulation than when administered alone.

In order to assess the effects of KLD in a multicellular system, more complex than established cell lines and closer to human model, we turned to the mouse model. While several studies addressed the question whether the use of omega- 3 or vitamin D or probiotics could be helpful to ameliorate colitis in humans and/or animal models (Barbalho et al., 2016; Claes et al., 2011; Ganji-Arjenaki and Rafieian-Kopaei, 2017; Hlavaty et al., 2015; Schwanke et al., 2016; Uranga et al., 2016), this is the first time that they have been administered together to assess anti-inflammatory effects in the gut. Accordingly, we administered KLD to C57BL/6 mice with a DSS-induced colitis. We found that treatment with KLD causes a remarkable reduction of acute colitis, in terms of recovery of body weight, clinical and histological scores and large intestine length. Besides, levels of proinflammatory cytokines, TNF- $\alpha$, IL- $1 \beta$ and IL-6, were significantly down-regulated in the inflamed tissues of KLD+DSS-treated mice as compared to DSStreated mice, while the anti-inflammatory cytokine IL-10 and VDR were significantly increased. Furthermore, given the importance of appropriate functioning of vitamin D and since VDR also functions as a transcription factor, we also used immunohistochemistry and found that VDR levels were strongly improved after KLD treatments in murine inflamed colon, suggesting its proper activation.

In conclusion, in vivo results confirm that KLD strongly reduces gut inflammation. All the results obtained from the study suggest that KLD would allow the patient with intestinal chronic inflammation to benefit from 
therapeutic coverage, even for very protracted periods, with no side effects. Certainly in vitro and in vivo models are not exhaustive and need further investigation in clinical trials. However if the results on man will confirm this data, this would open the horizon to a wider diffusion of this non-pharmacological treatment in patients with other immunological and also tumoural illnesses. Indeed, there are now a number of medical fields in which the correlation between immune regulatory activity, disease activity and prognosis has been demonstrated.

\section{Supplementary material}

Supplementary material can be found online at https://doi. org/10.3920/BM2017.0078.

Figure S1. (A) Effects of Lactobacillus reuteri (LR) and adhesive and invasive Escherichia coli strain LF82 on epithelial architecture (SF formation). (B) Vitality of LR in co-culture with CACO2 and HT29 in eukaryotic culture media, with or without KLD.

Figure S2. Effects of Lactobacillus reuteri on adhesion and invasion of Escherichia coli strain LF82.

Figure S3. Effect of KLD on mucosal healing evaluated by scratch test.

\section{Acknowledgements}

This work was supported by the Grant MIUR art.11 D.M. 593/2000 N.10/12 from Ministry of Education, University and Research (MIUR).

\section{References}

Albenberg, L.G. and Wu, G.D., 2014. Diet and the intestinal microbiome: associations, functions, and implications for health and disease. Gastroenterology 146: 1564-1572.

Barbalho, S.M., Goulart Rde, A., Quesada, K., Bechara, M.D. and De Carvalho Ade, C., 2016. Inflammatory bowel disease: can omega-3 fatty acids really help? Annals of Gastroenterology 29: 37-43.

Claes, I.J., De Keersmaecker, S.C., Vanderleyden, J. and Lebeer, S., 2011. Lessons from probiotic-host interaction studies in murine models of experimental colitis. Molecular Nutrition and Food Research 55: 1441-1453.

Costanzo, M., Cesi, V., Prete, E., Negroni, A., Palon, F., Cucchiara, S., Oliva, S., Leter, B. and Stronati, L., 2016. Krill oil reduces intestinal inflammation by improving epithelial integrity and impairing adherent-invasive Escherichia coli pathogenicity. Digestive and Liver Disease 48: 34-42.

Cruchet, S., Furnes, R., Maruy, A., Hebel, E., Palacios, J., Medina, F., Ramirez, N., Orsi, M., Rondon, L., Sdepanian, V., Xóchihua, L., Ybarra, M. and Zablah, RA., 2015. The use of probiotics in pediatric gastroenterology: a review of the literature and recommendations by Latin-American experts. Paediatric Drugs 17: 199-216.
Della Corte, C., Carpino, G., De Vito, R., De Stefanis, C., Alisi, A., Cianfarani, S., Overi, D., Mosca, A., Stronati, L., Cucchiara, S., Raponi, M., Gaudio, E., Byrne, C.D. and Nobili, V., 2016. Docosahexanoic acid plus vitamin D treatment improves features of NAFLD in children with serum vitamin D deficiency: results from a single centre trial. PLoS ONE 15: 11.

Dimitov, V. and White, J.H., 2017. Vitamin D signaling in intestinal innate immunity and homeostasis. Molecular Cell Endocrinology 15: 68-78.

Dolan, K.T. and Chang, E.B., 2016. Diet, gut microbes, and the pathogenesis of inflammatory bowel diseases. Molecular Nutrition and Food Research 61: 1.

Ganji-Arjenaki, M. and Rafieian-Kopaei, M., 2017. Probiotics are a good choice in remission of inflammatory bowel diseases: a meta-analysis and systematic review. Journal of Cellular Physiology 233: 2091-2103.

Grimm, V. and Riedel, C.U., 2016. Manipulation of the microbiota using probiotics. Advances in Experimental Medicine and Biology 902: 109-117.

He, B., Hoang, T.K., Wang, T., Ferris, M., Taylor, C.M., Tian, X., Luo, M., Tran, D.Q., Zhou, J., Tatevian, N., Luo, F., Molina, J.G., Blackburn, M.R., Gomez, T.H., Roos, S., Rhoads, J.M. and Liu, Y., 2017. Resetting microbiota by Lactobacillus reuteri inhibits $\mathrm{T}$ reg deficiency-induced autoimmunity via adenosine A2A receptors. Journal of Experimental Medicine 214: 107-123.

Hirata, Y., Ihara, S. and Koike, K., 2016. Targeting the complex interactions between microbiota, host epithelial and immune cells in inflammatory bowel disease. Pharmacological Research 113: 574-584.

Hlavaty, T., Krajcovicova, A. and Payer, J., 2015. Vitamin D therapy in inflammatory bowel diseases: who, in what form, and how much? Journal of Crohn's and Colitis 9: 198-209.

Hou, J.K., Abraham, B. and El-Serag, H., 2011. Dietary intake and risk of developing inflammatory bowel disease: a systematic review of the literature. American Journal of Gastroenterology 106: 563-573.

Huebner, C., Ding, Y., Petermann, I., Knapp, C. and Ferguson, L.R., 2011. The probiotic Escherichia coli Nissle 1917 reduces pathogen invasion and modulates cytokine expression in Caco-2 cells infected with Crohn's disease-associated E. coli LF82. Applied and Environmental Microbiology 77: 2541-2544.

Jiang, J., Li, K., Wang, F., Yang, B., Fu, Y., Zheng, J. and Li, D., 2016. Effect of marine-derived n-3 polyunsaturated fatty acids on major eicosanoids: a systematic review and meta-analysis from 18 randomized controlled trials. PLoS ONE 25: 11.

Kaliannan, K., Wang, B., Li, X.Y., Bhan, A.K. and Kang, J.X., 2016. Omega-3 fatty acids prevent early-life antibiotic exposure-induced gut microbiota dysbiosis and later-life obesity. International Journal of Obesity 40: 1039-1042.

König, J., Wells, J., Cani, P.D., García-Ródenas, C.L., MacDonald, T., Mercenier, A., Whyte, J., Troost, F. and Brummer, R.J., 2016. Human intestinal barrier function in health and disease. Clinical and Translational Gastroenterology 20: 7.

Kumari, M. and Kozyrskyj, A.L., 2017. Gut microbial metabolism defines host metabolism: an emerging perspective in obesity and allergic inflammation. Obesity Reviews 18: 18-31. 
Liu, Y., Fatheree, N.Y., Mangalat, N. and Rhoads, J.M., 2010. Humanderived probiotic Lactobacillus reuteri strains differentially reduce intestinal inflammation. American Journal of Physiology - Gastrointestinal and Liver Physiology 299: G1087-G1096.

Mangalat, N., Liu, Y., Fatheree, M.Y., Ferris, M.J., Van Arsdall, M.R., Chen, Z., Rahbar, M.H., Gleason, W.A., Norori, J., Tran, D.Q. and Rhoads, J.M., 2012. Safety and tolerability of Lactobacillus reuteri DSM 17938 and effects on biomarkers in healthy adults: results from a randomized masked trial. PLoS ONE 7: 43910.

Margulies, S.L., Kurian, D., Elliott, M.S. and Han, Z., 2015. Vitamin $D$ deficiency in patients with intestinal malabsorption syndromes - think in and outside the gut. Journal of Digestive Disease 16: 617-633.

Maxwell, J.R., Brown, W.A., Smith, C.L., Byrne, F.R. and Viney, J.L., 2009. Methods of inducing inflammatory bowel disease in mice. Current Protocols in Pharmacology 47: 5.58.1-5.58.37.

Meeker, S., Seamons, A., Maggio-Price, L. and Paik, J., 2016. Protective links between vitamin D, inflammatory bowel disease and colon cancer. World Journal of Gastroenterology 22: 933-948.

Mittal, R. and Coopersmith, C.M., 2014. Redefining the gut as the motor of clinical illness. Trends in Molecular Medicine 20: 214-223.

Odenwald, M.A. and Turner, J.R., 2017. The intestinal epithelial barrier: a therapeutic target? Nature Reviews Gastroenterology and Hepatology 14: 9-21.

Oliva, S., Di Nardo, G., Ferrari, F., Mallardo, S., Rossi, P., Patrizi, G., Cucchiara, S. and Stronati, L., 2012. Randomised clinical trial: the effectiveness of Lactobacillus reuteri ATCC 55730 rectal enema in children with active distal ulcerative colitis. Alimentary Pharmacology and Therapeutics 35: 327-334.

Rolhion, N. and Darfeuille-Michaud, A., 2007. Adherent-invasive Escherichia coli in inflammatory bowel disease. Inflammatory Bowel Disease 13: 1277-1283.

Sánchez, B., Delgado, S., Blanco-Míguez, A., Lourenço, A., Gueimonde, M. and Margolles, A., 2016. Probiotics, gut microbiota, and their influence on host health and disease. Molecular Nutrition and Food Research 61: 1.

Schwanke, R.C., Marcon, R., Bento, A.F. and Calixo, J.B., 2016. EPAand DHA-derived resolvins' actions in inflammatory bowel disease. European Journal Pharmacology 785: 156-164.

Shang, M. and Sun, J., 2017. Vitamin D/VDR, probiotics, and gastrointestinal diseases. Current Medicine Chemistry 24: 876-887.

Simopoulos, A.P., 2016. An increase in the omega-6/omega-3 fatty acid ratio increases the risk for obesity. Nutrients 8: 128 .

Tabbaa, M., Golubic, M., Roizen, M.F. and Bernstein, A.M., 2013. Docosahexaenoic acid, inflammation, and bacterial dysbiosis in relation to periodontal disease, inflammatory bowel disease, and the metabolic syndrome. Nutrients 5: 3299-3231.
Thomas, C.M., Saulnier, D.M., Spinler, J.K., Hemarajata, P., Gao, C., Jones, S.E., Grimm, A., Balderas, M.A., Burstein, M.D., Morra, C., Roeth, D., Kalkum, M. and Versalovic, J., 2016. FolC2-mediated folate metabolism contributes to suppression of inflammation by probiotic Lactobacillus reuteri. Microbiology Open 5: 802-818.

Uranga, J.A., López-Miranda, V., Lombó, F. and Abalo, R., 2016. Food, nutrients and nutraceuticals affecting the course of inflammatory bowel disease. Pharmacological Reports 68: 816-826.

Von Martels, J.Z., Sadaghian Sadabad, M., Bourgonje, A.R., Blokzijl, T., Dijkstra, G., Faber, K.N. and Harmsen, H.J., 2017. The role of gut microbiota in health and disease: in vitro modeling of host-microbe interactions at the aerobe-anaerobe interphase of the human gut. Anaerobe 44: 3-12.

Wall, R., Ross, R.P., Fitzgerald, G.F. and Stanton, C., 2010. Fatty acids from fish: the anti-inflammatory potential of long-chain omega-3 fatty acids. Nutrition Reviews 68: 280-289.

Wehkamp, J. and Frick, J.S., 2016. Microbiome and chronic inflammatory bowel diseases. Journal of Molecular Medicine 95: 21-28.

West, G.B. and Brown, J.H., 2005. The origin of allometric scaling laws in biology from genomes to ecosystems: towards a quantitative unifying theory of biological structure and organization. Journal of Experimental Biology 208: 1575-1592.

White, J.H., 2016. Vitamin D deficiency and the pathogenesis of Crohn's disease. Journal of Steroid Biochemistry and Molecular Biology 175: 23-28.

Wu, S., Liao, A., Xia, Y., Li, Y.C., Li, J.-D., Sartor, B. and Sun, J., 2010. Vitamin D receptor negatively regulates intestinal epithelial NFkappaB activity with bacterial stimulation. American Journal of Pathology 177: 686-697.

Wu, S., Yoon, S., Zhang, Y.G., Lu, R., Xia, Y., Wan, J., Petrof, E.O., Claud, E.C., Chen, D. and Sun, J., 2015. Vitamin D receptor pathway is required for probiotic protection in colitis. American Journal Physiology - Gastrointestinal Liver Physiology 309: G341-G349.

Ye, Y., Pang, Z., Chen, W., Ju, S. and Zhou, C., 2015. The epidemiology and risk factors of inflammatory bowel disease. International Journal of Clinical and Experimental Medicine 8: 22529-22542.

Zhang, Y., Wu, S. and Sun, J., 2013. Vitamin D, vitamin D receptor, and tissue barriers. Tissue Barriers 1: 1-6.

Zheng, B., Van Bergenhenegouwen, J., Van de Kant, H.J.G., Folkerts, G., Garssen, J., Vos, A.P., Morgan, M.E. and Kraneveld, A.D., 2016. Specific probiotic dietary supplementation leads to different effects during remission and relapse in murine chronic colitis. Beneficial Microbes 7: 205-213. 


$$
\gamma
$$

